\title{
Öffentlich-private Partnerschaften und kommunale Verschuldung
}

\author{
Berthold U. Wigger · Dominik H. Zimmermann
}

Online publiziert: 12. Mai 2020

(C) Der/die Autor(en) 2020

Zusammenfassung Der vorliegende Beitrag untersucht, ob es einen Zusammenhang gibt zwischen der Verschuldung einer Kommune und ihrer Neigung, Infrastrukturprojekte in Form von Öffentlich-privaten Partnerschaften zu realisieren. Ähnlich wie Kredite verschieben Öffentlich-private Partnerschaften (ÖPP) Zahlungsverpflichtungen der öffentlichen Hand in die Zukunft. Gelegentlich wird deshalb argumentiert, dass Gebietskörperschaften ÖPP dazu missbrauchen, das tatsächliche Ausmaß öffentlicher Verschuldung zu verschleiern. Kommunen, die das Verschuldungsinstrument bereits intensiv genutzt haben, sollten dann eine höhere ÖPP-Neigung aufweisen. Der vorliegende Beitrag findet dafür keine belastbare Evidenz. Mit Hilfe von Daten des Landes Nordrhein-Westfalen findet der Beitrag zwar, dass Kommunen mit ÖPP auch eine höhere Verschuldung aufweisen. In einer ökonometrischen Analyse entpuppt sich der Effekt der Verschuldung auf die Anzahl von ÖPP aber als insignifikant und zudem quantitativ unbedeutend.

Schlüsselwörter Öffentlich-private Partnerschaften · kommunale Verschuldung · fiskalische Regeln

JEL classification $\mathrm{H} 54 \cdot \mathrm{H} 72 \cdot \mathrm{H} 74$ 


\title{
Public-private partnerships and municipal debt
}

\begin{abstract}
The present paper examines whether there is a connection between the debt of a municipality and its tendency to implement infrastructure projects in the form of public-private partnerships. Similar to loans, public-private partnerships (PPPs) postpone public sector payment obligations into the future. It is therefore sometimes argued that local authorities misuse PPPs to disguise the true level of public debt. Municipalities that have already used the debt instrument intensively should then have a higher PPP tendency. The present paper does not find any reliable evidence for this. Employing data of the state of North Rhine-Westfalia the paper finds that municipalities with PPPs do in fact have higher levels of debt. In an econometric analysis, however, the effect of debt on the number of PPPs turns out to be insignificant and also quantitatively negligible.
\end{abstract}

Keywords Public-private partnership · Municipal Debt · Fiscal restraint

\section{Einleitung}

In Öffentlich-privaten Partnerschaften (ÖPP) erstellen und betreiben private Partner der öffentlichen Hand nicht nur die Infrastruktur, sondern beteiligen sich oft auch an deren Finanzierung. ${ }^{1}$ Für die Bereitstellung der Infrastruktur erhält der private Partner während der Betriebsphase vom öffentlichen Partner entsprechende Entgelte. Deshalb werden in ÖPP Finanzierungsverpflichtungen des Staates ähnlich wie bei öffentlichen Krediten in die Zukunft verschoben. Bei öffentlichen Krediten müssen in der Zukunft Zinsen und Tilgung geleistet werden, in ÖPP müssen Staat oder Steuerzahler in Zukunft Entgelte für die Infrastruktur an den privaten Partner zahlen.

Anders als öffentliche Kredite müssen die Finanzierungsverpflichtungen des Staates in ÖPP aber gegebenenfalls nicht als öffentliche Verschuldung ausgewiesen werden. Deshalb mögen Gebietskörperschaften einen Anreiz haben, Infrastrukturprojekte in ÖPP zu realisieren, statt sie mit Krediten zu finanzieren. Das gilt insbesondere dann, wenn der Verschuldungsspielraum der Gebietskörperschaften beschränkt ist, beispielsweise weil bereits ein hohes Verschuldungsniveau erreicht ist oder weil die Verschuldung einer fiskalischen Regel wie der Schuldenbremse unterliegt. In solchen Situationen hat die öffentliche Hand einen Anreiz, das tatsächliche Ausmaß künftiger Finanzierungslasten zu verschleiern (Easterly 1999). So wurde wiederholt argumentiert, dass ÖPP insbesondere vor dem Hintergrund gesetzlicher Verschuldungsregeln Gebietskörperschaften die Möglichkeit geben, künftige Zahlungsverpflichtungen außerhalb der konventionellen Kreditaufnahme einzugehen (Auriol und Picard 2013; Funke et al. 2013; Mühlenkamp 2014).

Deutsche Kommunen haben formal nur einen relativ geringen Verschuldungsspielraum. So dürfen sie Kredite in der Regel nur für Investitionen oder zur Umschuldung aufnehmen. ${ }^{2}$ Neben Investitionskrediten haben die Kommunen jedoch

\footnotetext{
1 Im Detail siehe Wigger (2017).

2 Siehe z. B. Gemeindeordnung für das Land Nordrhein-Westfalen (GO NRW) $§ 86$.
} 
auch die Möglichkeit, zur Sicherung der eigenen Liquidität Kassenkredite aufzunehmen. Mithilfe dieses Instruments kann die klassische Investitionsbindung kommunaler Kredite faktisch umgangen werden. Etwa seit dem Beginn der 90er-Jahre verschulden sich insbesondere nordrhein-westfälische Kommunen zunehmend in Form von Kassenkrediten. ${ }^{3}$ Kommunen ist es zudem in der Regel nicht erlaubt sich zu überschulden. ${ }^{4}$ Gleichwohl wird auch dies nicht von allen Kommunen eingehalten. Um die Einhaltung der Verschuldungsregeln durchzusetzen, wurden die kommunalen Aufsichtsbehörden deshalb mit zunehmend restriktiveren Kontrollmöglichkeiten ausgestattet. Ein wesentliches Instrument ist die Verpflichtung von Kommunen zur Vorlage von Haushaltssicherungskonzepten (HSK). Seit 2011 sind überschuldete Kommunen in Nordrhein-Westfalen zudem zur Teilnahme an einem landesweiten Konsolidierungsprogramm, dem sogenannten „Stärkungspakt Stadtfinanzen“, verpflichtet. Die Verwendung dieser Instrumente kann für die betroffenen Kommunen tiefgreifende Eingriffe in ihre Haushaltsautonomie bedeuten. ${ }^{5}$ Daher könnte man annehmen, dass hohe Verschuldungsniveaus gerade hier Anreize zur Verschleierung der tatsächlichen Verschuldung auslösen.

In anderen Ländern konnte ein Zusammenhang zwischen der Verschuldung staatlicher Institutionen und ihrer Neigung, Infrastrukturprojekte als ÖPP zu realisieren, nachgewiesen werden. Albalate et al. (2015) liefern Evidenz dafür, dass Infrastrukturprojekte auf US-Staaten-Ebene mit einer stärkeren Übertragung von Risiken an private Unternehmen, u. a. in Form von ÖPP, einhergehen, wenn die Staaten eine höhere Verschuldung aufweisen. Auf europäischer Ebene finden Russo und Zampino (2010) für Italien und Buso et al. (2017) für Frankreich einen signifikanten Zusammenhang zwischen der Verschuldung von Kommunen und der Anzahl von ÖPP-Projekten, wobei letztere allerdings nur einen Zusammenhang für den Schuldenstand, nicht aber für die Nettokreditaufnahme nachweisen.

Für Deutschland liegt bislang keine vergleichbare Untersuchung vor. Der vorliegende Beitrag versucht, diese Lücke zu schließen. Er analysiert, inwieweit Kommunen mit höherer Verschuldung stärker dazu neigen, ÖPP einzugehen als Kommunen mit geringerer Verschuldung. Eine empirische Untersuchung von ÖPP in Deutschland steht freilich vor dem Problem einer sehr mangelhaften Datenlage. Zwar werden ÖPP in der PPP-Projektdatenbank der Partnerschaften Deutschland erfasst, zudem enthält die kommunale Schuldenstatistik Informationen über ÖPP-Projekte, beide Datenbanken sind aber unvollständig.

Ein erster Schritt der vorliegenden Studie besteht deshalb darin, aus den verfügbaren Datenquellen einen belastbaren Datensatz für die empirische Analyse zu entwickeln. Auf kommunaler Ebene wurden die meisten ÖPP im Land NordrheinWestfalen eingegangen. Der für die vorliegende Studie entwickelte Datensatz be-

\footnotetext{
3 Bei einem Anteil an der Gesamtbevölkerung von ca. $22 \%$ entfielen im Jahr 2018 rund $63 \%$ der Kassenkredite der Kernhaushalte der Gemeinden und Gemeindeverbände beim nicht-öffentlichen Bereich auf Nordrhein-Westfalen (Destatis 2019).

${ }^{4}$ Siehe z. B. GO NRW $§ 75$. In der Doppik gilt eine Gemeinde als überschuldet, wenn nach der Bilanz das Eigenkapital aufgebraucht ist (vgl. GO NRW § 75 (1)).

5 Für eine detaillierte Darstellung siehe Rappen (2017).
} 
rücksichtigt alle nordrhein-westfälischen Kommunen ${ }^{6}$ und untersucht konkret, ob höher verschuldete Kommunen mit größerer Häufigkeit ÖPP eingehen. Neben der vergleichsweise großen Anzahl an ÖPP-Projekten bietet die Fokussierung auf Nordrhein-Westfalen einige weitere Vorteile. Erstens unterliegen alle betrachteten Kommunen dem gleichen kommunalen Regulierungsrahmen, da das kommunale Recht in Deutschland Ländersache ist. Zweitens liegt in Nordrhein-Westfalen starke Variation in den kommunalen Verschuldungsniveaus vor. Drittens muss in NordrheinWestfalen anders als in manchen anderen Bundesländern, beispielsweise BadenWürttemberg, nicht zwischen Gemeinden und Gemeindeverbänden unterschieden werden. Im Ergebnis stellen die nordrhein-westfälischen Kommunen relativ gut miteinander vergleichbare Betrachtungseinheiten dar, die sich gleichwohl in den für die vorliegende Studie zentralen Merkmalen - Verschuldungsneigung und eingegangene ÖPP - hinreichend voneinander unterscheiden.

Eine deskriptive Auswertung der Daten unterstützt die Vermutung, dass höher verschuldete Kommunen stärker dazu neigen, Infrastrukturprojekte in ÖPP zu realisieren. Das gilt in besonderem Maße für Kommunen, die von der Kommunalaufsicht des Landes als überschuldet eingestuft worden sind. Indessen lässt sich dieser Zusammenhang nicht durch eine Regressionsanalyse bestätigen. Es zeigt sich, dass die Häufigkeit, mit der eine Kommune ÖPP-Projekte eingeht, viel besser durch andere fiskalische Größen und durch demografische Variablen erklärt werden kann. Im Ergebnis findet die vorliegende Studie keine empirische Evidenz für den vermuteten Zusammenhang zwischen ÖPP-Projekten und kommunaler Verschuldung.

Der weitere Gang der Untersuchung gliedert sich wie folgt: Abschn. 2 entwickelt den der empirischen Analyse zugrundeliegenden Datensatz. Abschn. 3 enthält eine deskriptive Analyse des Datensatzes sowie eine Regressionsanalyse. Abschn. 4 schließt mit einem kurzen Fazit.

\section{Daten zu kommunalen ÖPP in Deutschland}

\subsection{Datenquellen}

Ein wesentliches Problem jedweder Untersuchung zu ÖPP in Deutschland ist die unzureichende Verfügbarkeit von geeigneten Daten. Bereits die genaue Anzahl an Projekten ist unbekannt. Zwar existiert eine ganze Reihe von möglichen Datenquellen, jedoch sind diese unvollständig. Insbesondere gibt es keine amtliche Erhebung mit dem Anspruch, alle ÖPP-Projekte in Deutschland zu erfassen. Existierende Erhebungen basieren entweder auf freiwilligen Angaben oder erfassen ÖPP-Projekte nur, sofern sie bestimmte Charakteristika aufweisen. In der vorliegenden Untersuchung wird primär auf zwei Quellen zurückgegriffen, welche für eine empirische

\footnotetext{
6 Die 2009 neugebildete Städteregion Aachen stellt einen Gemeindeverband besonderer Art dar, der verschiedene Besonderheiten hinsichtlich der kommunalen Aufgabenverteilung aufweist. Die Gemeinden der Region wurden deshalb in der späteren empirischen Analyse nicht berücksichtigt.
} 
Analyse noch am besten geeignet erscheinen. ${ }^{7}$ Hierbei handelt es sich um die PPPProjektdatenbank (Partnerschaft Deuschtschland 2020) sowie die ÖPP-relevanten Finanzdaten der kommunalen Schuldenstatistik. Weitere Quellen wie etwa Haushaltspläne, Vergabebekanntmachungen und Medienberichte wurden zur Validierung und Ergänzung des Datensatzes herangezogen.

Die von der Partnerschaft Deutschland ${ }^{8}$ verwaltete PPP-Projektdatenbank stellt aktuell die umfangreichste Datenbank deutscher ÖPP-Projekte dar. Sie wird daher häufig zur Quantifizierung und Untersuchung von ÖPP in Deutschland verwendet (u. a. ÖPP Deutschland AG 2011; Hesse et al. 2013). Ein wesentliches Problem der Datenbank ist jedoch, dass eine Eintragung von Projekten nur auf freiwilliger Basis erfolgt und sie daher unvollständig ist.

Bis Anfang 2020 wurden in der PPP-Projektdatenbank deutschlandweit Datensätze zu insgesamt 266 Hochbau- und Verkehrsprojekten geführt. Dabei handelt es sich nach Angaben der PD um 27 Projekte in der Ausschreibung und 239 Projekte mit Vertragsabschluss (Partnerschaft Deuschtschland 2020). Unter den Projektträgern finden sich dabei sowohl Bund und Länder als auch Gemeindeverbände und Gemeinden.

Von den Projekten mit Vertragsabschluss lassen sich bis zu 143 Projekte der Gemeindeebene zuordnen, wobei sich nicht mit Gewissheit sagen lässt, ob alle in der PPP-Projektdatenbank aufgelisteten Projekte tatsächlich realisiert werden oder wurden. In der PPP-Projektdatenbank sind nämlich mehrere „Projekte mit Vertragsschluss“ zu finden, zu denen es keinerlei weitere Informationen gibt, die bestätigen, dass es tatsächlich zu einem Vertragsschluss gekommen ist. Vertragsabschlüsse konnten mehrfach nicht durch andere Quellen bestätigt werden. Daher ist anzunehmen, dass in der Datenbank mehrfach „Projekte mit Vertragsschluss“ geführt werden, die tatsächlich nicht als ÖPP umgesetzt wurden.

Wie in Tab. 1 dargestellt, existieren zwischen den einzelnen Bundesländern erhebliche Unterschiede hinsichtlich der Projektanzahl. In Nordrhein-Westfalen wurde die mit Abstand größte Anzahl an ÖPP eingegangen. Dort konnten 56 ÖPP-Projekte auf Gemeindeebene identifiziert werden, für die erkennbar ein Vertrag abgeschlossen wurde. Bundesweit entspricht das $39 \%$ aller ÖPP-Projekte auf Gemeindeebene. Die spätere empirische Analyse untersucht ÖPP-Projekte in Nordrhein-Westfalen. Deshalb wurden für dieses Bundesland sämtliche Projekte dahingehend überprüft, ob

\footnotetext{
7 Daneben stellen auch die Haushalts- und Finanzplanung öffentlicher Gebietskörperschaften, Berichte von Rechnungshöfen und Rechnungsprüfämtern sowie Vergabebekanntmachungen mögliche Informationsquellen dar. Mögliche weitere nichtamtliche Datenquellen zu ÖPP sind zudem Projektlisten der Bau-, Finanz- und Beratungsindustrie, kommerzielle Nachrichtendienste sowie die Datenbank des European PPP Expertise Centers (EPEC). Diese Quellen haben jedoch gemein, dass sie weder hinsichtlich des Umfangs noch der Qualität der Daten mit der PPP-Projektdatenbank und der Schuldenstatistik vergleichbar sind.

8 Die Partnerschaft Deutschland entstand 2016 durch die Umwandlung der 2008 gegründeten ÖPP Deutschland AG. Ursprünglich wurde die ÖPP Deutschland AG als gemischtwirtschaftliches Unternehmen mit dem Ziel gegründet, öffentliche Körperschaften bei der Planung und Umsetzung von ÖPP zu beraten. Seit der Umwandlung ist das Unternehmen unter Beteiligung von Bund, Ländern und Kommunen vollständig in öffentlicher Hand. Die Partnerschaft Deutschland soll nun unabhängig davon, ob eine ÖPP in Betracht kommt, öffentliche Körperschaften bei Beschaffungsvorhaben beraten. Das Unternehmen ist darüber hinaus mit Grundlagen- und Öffentlichkeitsarbeit zu ÖPP beauftragt. Unter anderem zählt hierzu auch die Verwaltung der PPP-Projektdatenbank.
} 
Tab. 1 Anzahl an ÖPP-Projekten je Bundesland (Stand Februar 2020). (Eigene Darstellung; Datenquelle: PPP-Projektdatenbank)

\begin{tabular}{lll}
\hline Bundesland & $\begin{array}{l}\text { Projekte insgesamt mit und } \\
\text { ohne Vertragsabschluss }\end{array}$ & $\begin{array}{l}\text { Davon Gemeindeprojekte mit } \\
\text { Vertragsabschluss }\end{array}$ \\
\hline Baden-Württemberg & 26 & 7 \\
Bayern & 34 & 11 \\
Berlin & 2 & - \\
Brandenburg & 13 & 7 \\
Bremen & 1 & - \\
Hamburg & 3 & - \\
Hessen & 31 & 10 \\
Mecklenburg-Vorpommern & 2 & 1 \\
Niedersachsen & 24 & 11 \\
Nordrhein-Westfalen & 73 & 56 \\
Rheinland-Pfalz & 3 & 3 \\
Saarland & 3 & 2 \\
Sachsen & 6 & 3 \\
Sachsen-Anhalt & 16 & 13 \\
Schleswig-Holstein & 24 & 18 \\
Thüringen & 5 & 1 \\
Summe & 266 & 143 \\
\hline
\end{tabular}

sie durch weitere Quellen bestätigen lassen. Bei den in Tab. 1 angegebenen 56 ÖPPProjekten auf Gemeindeebene handelt es sich daher ausschließlich um verifizierte Projekte mit Vertragsabschluss. Die angegebenen Werte für die übrigen Bundesländer können dagegen die tatsächlichen Projektzahlen überschätzen und sind deshalb als obere Grenzen anzusehen. Tatsächlich dürften sogar mehr als $39 \%$ aller ÖPPProjekte auf Gemeindeebene in Nordrhein-Westfalen umgesetzt werden.

Die öffentliche Schuldenstatistik ist bisher die einzige bundesweite amtliche Statistik mit Daten zu ÖPP. Seit dem Haushaltsjahr 2010 werden dort Zahlungsverpflichtungen für laufende ÖPP erfasst, welche gemäß dem Europäischen System Volkswirtschaftlicher Gesamtrechnungen (ESVG) dem öffentlichen Sektor zuzurechnen sind. ${ }^{9}$ Seit dem Haushaltsjahr 2012 erfolgt zudem eine getrennte Aufschlüsselung nach bereits geleisteten Zahlungen ${ }^{10}$. Zielsetzung ist es dabei, dass ÖPP im Rahmen der für die Überprüfung der Einhaltung der Kriterien des europäischen Stabilitäts- und Wachstumspaktes relevanten Volkswirtschaftlichen Gesamtrechnung (VGR) korrekt dem öffentlichen oder dem privaten Sektor zugerechnet werden. Dies soll unter anderem verhindern, dass die öffentliche Verschuldung mittels ÖPP ver-

\footnotetext{
9 Eine ÖPP ist gemäß ESVG 2010 dem öffentlichen Bereich zuzuordnen, sofern nicht das Baurisiko und das Verfügbarkeits- oder Nachfragerisiko an den privaten Partner übertragen wurde (Eurostat 2014, S. $522 \mathrm{ff})$.

10 Im Rahmen der Schuldenstatistik werden dabei jedoch nur Verpflichtungen erfasst, die dem öffentlichen Gesamthaushalt zurechenbar sind. Nicht erfasst werden daher insbesondere Projekte, die öffentlichen Fonds, Einrichtungen und Unternehmen zuzuordnen sind.
} 
Tab. 2 Gemeinden mit ÖPP in Nordrhein-Westphalen nach Datenquelle. (Eigene Berechnungen; Datenquelle: PPP-Projektdatenbank (Partnerschaft Deuschtschland 2020) und Statistisches Landesamt NRW (2016))

\begin{tabular}{lll}
\hline & $\begin{array}{l}\text { Anzahl Ge- } \\
\text { meinden }\end{array}$ & $\begin{array}{l}\text { Anteil an den insgesamt identi- } \\
\text { fizierten Gemeinden (in \%) }\end{array}$ \\
\hline Projektdatenbank & 42 & 91,3 \\
Schuldenstatistik & 28 & 60,9 \\
Nur Projektdatenbank & 18 & 39,1 \\
Nur Schuldenstatistik & 4 & 8,7 \\
Projektdatenbank \& Schuldenstatistik & 24 & 52,3 \\
Insgesamt & 46 & - \\
\hline
\end{tabular}

schleiert werden kann. In der Schuldenstatistik wird also nur eine Teilmenge aller mit ÖPP in Verbindung stehenden Verpflichtungen ausgewiesen, nämlich diejenigen, bei denen nur in geringem Umfang Risiken an den privaten Sektor übertragen wurden.

Anhand der in der Schuldenstatistik ausgewiesenen Verpflichtungen lassen sich Kommunen identifizieren, welche ÖPP-Projekte durchführen. In Nordrhein-Westfalen wurden auf diese Weise insgesamt 31 Gemeinden mit ÖPP ermittelt. Im Gegensatz zur PPP-Projektdatenbank lassen sich jedoch anhand der summierten Verpflichtungen keine individuellen Projekte identifizieren. Die öffentliche Schuldenstatistik ist daher für sich genommen ungeeignet, um ÖPP-Projekte zu quantifizieren. Hinzu kommt, dass entsprechende Daten dort erst ab dem Jahr 2010 verfügbar sind. Für die weitere Analyse wurden daher, soweit möglich, die in der Schuldenstatistik aufgeführten Verpflichtungen mittels zusätzlicher Quellen, vor allem Haushaltspläne, PPP-Projektdatenbank, Vergabebekanntmachungen und Medienberichte, konkreten ÖPP-Projekten zugeordnet.

\subsection{Abgleich von Projektdatenbank und Schuldenstatistik}

Weil sowohl die PPP-Projektdatenbank als auch die öffentliche Schuldenstatistik jeweils nur eine Teilmenge der tatsächlich durchgeführten ÖPP-Projekte enthalten, wurde im Rahmen der vorliegenden Analyse mittels Kreuzvalidierung die Güte der beiden Datensätze evaluiert und zugleich ein möglichst umfassendes Bild für ÖPP-Projekte in Nordrhein-Westfalen gewonnen. Mithilfe der PPP-Projektdatenbank konnten 56 ÖPP-Projekte mit Vertragsabschluss in Gemeinden und kreisfreien Städten identifiziert werden. ${ }^{11}$ Diese Projekte verteilen sich dabei auf insgesamt 42 Kommunen. Über die Schuldenstatistik konnten anhand entsprechender Verpflichtungen vier weitere Kommunen mit ÖPP-Projekten identifiziert werden, für die keine entsprechenden Projekte in der PPP-Projektdatenbank aufgeführt sind (siehe Tab. 2).

Über die Schuldenstatistik sind bei weitem nicht alle in der PPP-Projektdatenbank geführten Projekte identifizierbar. Dies kann beispielsweise mit der eingeschränkten

11 Projekte auf Ebene der Landkreise wurden im Rahmen der weiteren Analyse nicht untersucht. 


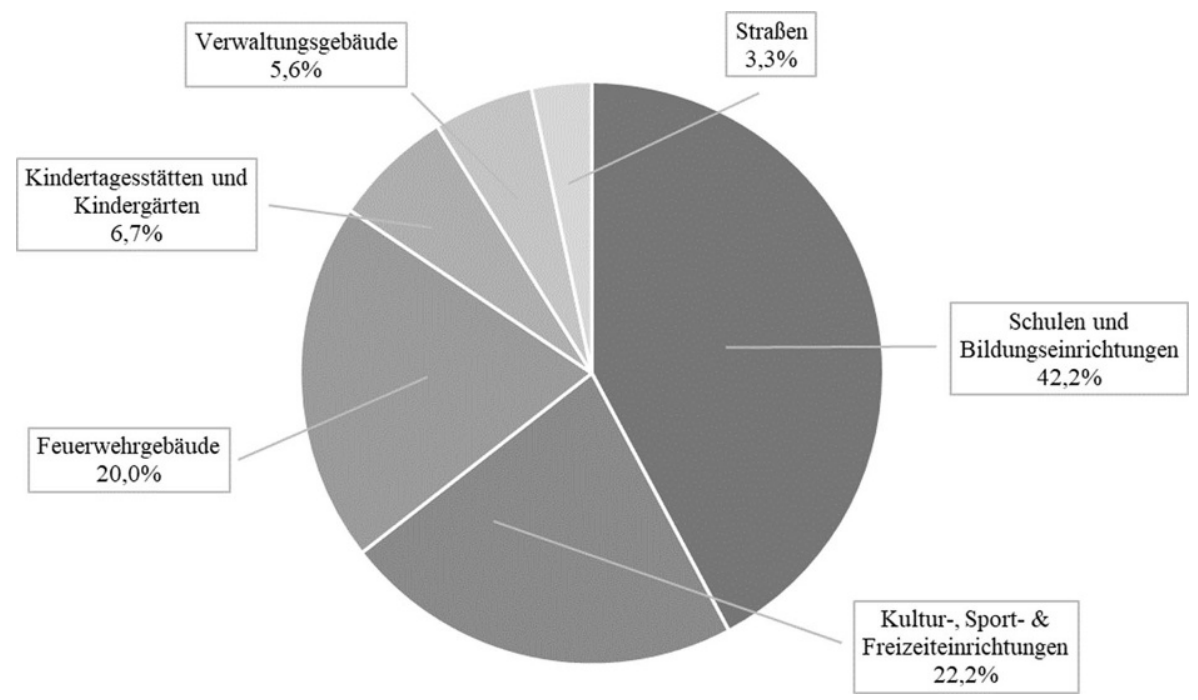

Abb. 1 Nordrhein-westfälische ÖPP-Projekte auf Gemeindeebene nach Projektart (Basierend auf 60 identifizierten Projekten. Bei Projekten, die mehreren Kategorien zugeordnet werden konnten, ist die Zuordnung anteilig erfolgt)

ÖPP-Definition gemäß ESVG (Eurostat 2014) zusammenhängen. Möglich ist aber auch, dass die Projekte über kommunale Unternehmen gehalten werden und folglich nicht im Haushalt der Kommunen auftauchen. Der direkte Vergleich zeigt, dass die PPP-Projektdatenbank wesentlich vollständiger ist (91,3\% der Kommunen) als die Schuldenstatistik (60,9\% der Kommunen). Gleichwohl bietet die PPP-Projektdatenbank allein kein umfassendes Bild der Bedeutung von ÖPP.

Durch die Hinzunahme von vier zusätzlichen ÖPP-Projekten, für die in der Schuldenstatistik entsprechende Verpflichtungen ausgewiesen sind und die sich durch weitere Quellen identifizieren lassen, ergibt sich eine Gesamtanzahl von 60 ÖPPProjekten. Um welche Art von Projekten es sich dabei handelt, ist in Abb. 1 dargestellt. Den größten Anteil haben mit 42,2\% Schulen und Bildungseinrichtungen. Hohe Anteile entfallen zudem auf Kultur-, Sport- und Freizeiteinrichtungen sowie Feuerwehrgebäude mit 22,2 und 20,0\%. Bei den übrigen 15,6\% der Projekte handelt es sich um Kindertagesstätten und Kindergärten, Verwaltungsgebäude sowie Straßenbauprojekte.

Insgesamt entsteht durch die Zusammenführung beider Datensätze ein vollständigeres Bild der quantitativen Bedeutung von ÖPP in Nordrhein-Westfalen. Jedoch ist aufgrund der Freiwilligkeit der Eintragung in der PPP-Projektdatenbank und der obligatorischen, aber unvollständigen Erfassung von Projekten im Rahmen der kommunalen Schuldenstatistik nicht auszuschließen, dass ÖPP existieren, welche in keiner der beiden Datenbanken aufgeführt sind. Die genaue Anzahl an ÖPPProjekten lässt sich mit anderen Worten nicht mit Sicherheit ermitteln. 
Abb. 2 Nordrhein-westfälische Gemeinden mit und ohne ÖPP (Gemeinden mit ÖPP-Projekten sind dunkelgrau dargestellt, alle übrigen Gemeinden hellgrau)

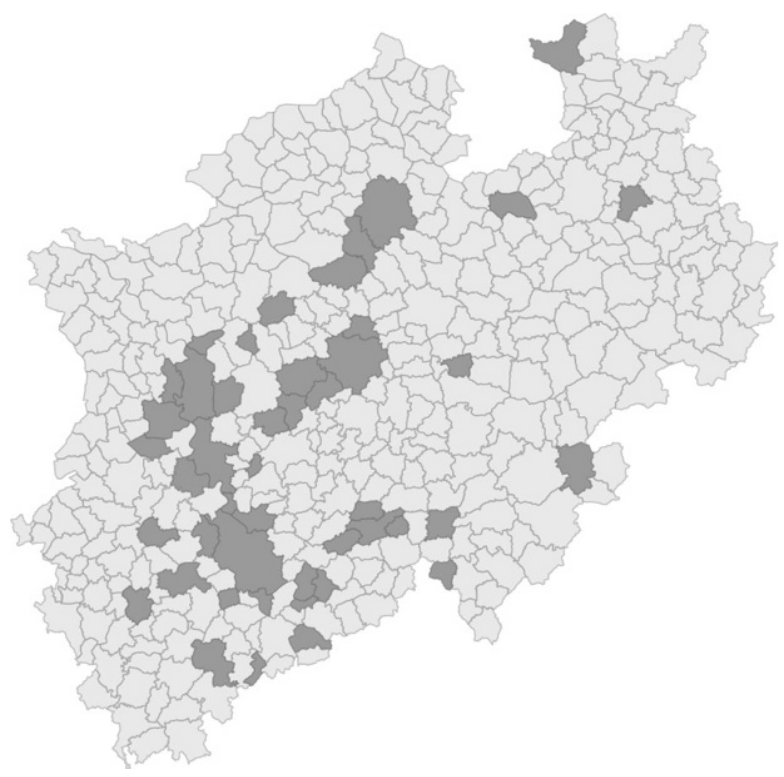

\section{Empirische Analyse}

\subsection{Deskriptive Auswertung}

In die empirische Analyse gehen Daten zu 386 nordrhein-westfälischen Kommunen aus dem Zeitraum zwischen 2010 und 2015 ein, die zu einem Querschnittsdatensatz zusammengefasst wurden. Für weiter zurückliegende Perioden sind die entsprechenden Daten zum Teil nicht verfügbar. Die Daten zu ÖPP entstammen wie im vorangegangen Kapitel beschrieben der kommunalen Schuldenstatistik und der PPP-Projektdatenbank. Daten zur kommunalen Verschuldung und zu weiteren kommunalen Kennzahlen wurden neben der Schuldenstatistik der Regionaldatenbank Deutschland (Statistische Ämter 2019) sowie den Veröffentlichungen des Landeswahlleiters (Landeswahlleiter 2014) entnommen.

Die räumliche Verteilung der untersuchten Kommunen ist in Abb. 2 dargestellt. Der Datensatz setzt sich aus den 46 in Abschn. 2 ermittelten Kommunen zusammen, in denen ÖPP-Projekte durchgeführt wurden, und weiteren 340 Kommunen ohne entsprechende Projekte.

Eine deskriptive Analyse der verfügbaren Daten zeigt, dass sich die beiden Kommunengruppen deutlich voneinander unterscheiden (siehe Tab. 3). Insbesondere existieren größere Unterschiede hinsichtlich der Verschuldung. Kommunen mit ÖPPProjekten weisen eine deutlich höhere Pro-Kopf-Verschuldung auf als Kommunen ohne ÖPP-Projekte. Im Mittel ist die Pro Kopf-Verschuldung in ÖPP-Kommunen rund $65 \%$ höher (bei Investitionskrediten $62 \%$, bei Kassenkrediten 69\%). Auch die Nettokreditaufnahme ist in ÖPP-Kommunen im Mittel um 70\% höher. Außer- 
Tab. 3 Vergleich der Gruppen hinsichtlich fiskalischer und struktureller Rahmendaten

\begin{tabular}{lllll}
\hline & Mit ÖPP & & Ohne ÖPP & Median \\
\hline Verschuldung [t $€$ pro Kopf] & Mittelwert & Median & Mittelwert & M,95 \\
Investitionskredite [t $€$ pro Kopf] & 2,03 & 1,99 & 1,23 & 0,60 \\
Kassenkredite [t $€$ pro Kopf] & 1,10 & 1,02 & 0,68 & 0,23 \\
Nettokreditaufnahme [t $€$ pro Kopf] & 0,93 & 0,50 & 0,55 & 0,03 \\
Überschuldet [t $€$ pro Kopf] & 0,08 & 0,05 & 0,04 & 0 \\
Einwohner & 0,17 & 0 & 0,06 & 19.679 \\
Fläche [km ${ }^{2}$ ] & 124.511 & 50.580 & 34.071 & 74,66 \\
Einwohnerdichte [EW pro km ${ }^{2}$ ] & 100,50 & 76,11 & 84,66 & 257 \\
Kreisfreie Stadt & 1034 & 861 & 428 & 0 \\
BIP auf Kreisebene [t $€$ pro Kopf] & 0,20 & 0 & 0,04 & 30,05 \\
Steuereinnahmekraft [t $€$ pro Kopf] & 34,24 & 33,67 & 31,20 & 0,98 \\
Stimmanteil CDU/FDP [\%] & 1,075 & 0,958 & 1,301 & 48,4 \\
$N$ & 42,9 & 44,2 & 48,3 & 340 \\
\hline
\end{tabular}

Bei allen in Euro angegebenen Werten handelt es sich um Mittelwerte der Jahre 2011-2015

Die Stimmanteile von CDU und FDP beziehen sich auf die abgegebenen Stimmen bei den Stadt- und Gemeinderatswahlen 2014

dem werden ÖPP-Kommunen mit einer dreimal höheren Häufigkeit als überschuldet eingestuft als Kommunen ohne ÖPP. ${ }^{12}$

Höhere Pro-Kopf-Verschuldung, höhere Pro-Kopf-Nettokreditaufnahme und höhere Überschuldungshäufigkeit von ÖPP-Kommunen legen nahe, dass in der Tat ein positiver Zusammenhang besteht zwischen der Verschuldung einer Kommune und ihrer Neigung Infrastrukturprojekte in ÖPP zu realisieren. Jedoch unterscheiden sich Kommunen mit und ohne ÖPP auch hinsichtlich anderer Variablen, die einen Einfluss auf ihre ÖPP-Neigung haben mögen, zum Teil erheblich. ÖPP-Kommunen sind mit rund 124.000 Einwohnern im Mittel deutlich größer als die übrigen Kommunen mit durchschnittlich 34.000 Einwohnern. Die Bevölkerungsdichte ist im Durchschnitt fast 2,5-mal so groß. Zudem handelt es sich bei $20 \%$ der ÖPPKommunen um kreisfreie Städte, bei den Kommunen ohne ÖPP sind es nur $4 \%$. ÖPP-Kommunen weisen außerdem mit einer $17 \%$ niedrigeren Steuereinnahmekraft eine geringere finanzielle Basis auf. Auch hinsichtlich des Wahlverhaltens ihrer Bürger weisen die Kommunen deutliche Unterschiede auf. Bei der Kommunalwahl 2014 erzielten bürgerliche Parteien in Gemeinden mit ÖPP um 5,4 Prozentpunkte schlechtere Ergebnisse als in Gemeinden ohne ÖPP. Schließlich existieren, wenn auch weniger große, Unterschiede bei Fläche und Bruttoinlandsprodukt (BIP) pro Kopf. ${ }^{13}$

\footnotetext{
12 Kommunen werden in der vorliegenden Untersuchung als überschuldet eingestuft, wenn sie im Haushaltsjahr 2017 negatives Eigenkapital aufwiesen (siehe Ministerium für Inneres und Kommunales NRW 2018).

13 Das BIP wird nur auf Kreisebene ermittelt. Die gemeindespezifische Werte der kreisangehörigen Gemeinden beziehen sich daher jeweils auf die Werte für den gesamten Landkreis.
} 
Ob eine Kommune ein Infrastrukturprojekt als ÖPP umsetzt, hängt natürlich auch von ihrem Infrastrukturbedarf ab, denn bei höherem Bedarf werden tendenziell mehr Projekte umgesetzt, so dass die Wahrscheinlichkeit steigt, dass sich darunter ÖPPProjekte befinden. Kommunen mit einer höheren Einwohnerzahl haben auch einen höheren Infrastrukturbedarf. Darüber hinaus wird allgemein davon ausgegangen, dass der Bedarf nicht nur mit der Bevölkerung wächst, sondern insbesondere auch mit steigender Bevölkerungsdichte. ${ }^{14}$ So werden in Ballungszentren tendenziell mehr und höherwertigere öffentliche Leistungen nachgefragt. Gleichzeitig erbringen Ballungszentren auch zentralörtliche Leistungen für das Umland. Zudem ist die Bereitstellung öffentlicher Leistungen in Ballungszentren insbesondere aufgrund höherer Grundstückspreise häufig mit höheren Kosten verbunden. Die kreisfreien Städte nehmen hierbei eine Sonderrolle ein, da sie zusätzlich zum Aufgabenspektrum der kreisangehörigen Gemeinden auch die Aufgaben einer Kreisverwaltung erfüllen. Hierzu zählt, unter anderem die Zuständigkeit für Krankenhäuser, den öffentlichen Personennahverkehr, die Kreisstraßen, die Abfallbeseitigung sowie die Berufs- und Sonderschulen. Folglich unterscheiden sich ihre Infrastrukturanforderungen nach Umfang und Art von jenen der kreisangehörigen Gemeinden. Darüber hinaus besteht auch ein enger Zusammenhang zwischen der Entwicklung der Wirtschaftskraft, den Steuereinnahmen und der Bereitstellung öffentlicher Leistungen. Schließlich gibt es Hinweise darauf, dass individuelle Werthaltungen einen starken Einfluss darauf ausüben, ob eine Personen ÖPP gutheißt oder nicht. ${ }^{15}$ So scheinen Anhänger bürgerlicher Parteien wie CDU und FDP ÖPP grundsätzlich eher zu favorisieren als Anhänger stärker etatistischer Parteien wie SPD und Grüne. Deshalb mag auch der Anteil bürgerlicher Parteien in den kommunalen Vertretungen einen Einfluss darauf haben, in welchem Umfang Infrastrukturprojekte als ÖPP umgesetzt werden.

Insgesamt legt eine deskriptive Auswertung der Daten nahe, dass vor allem stärker verschuldete Kommunen ÖPP eingehen. Allerdings handelt es sich dabei auch um bevölkerungsreichere und urbanere Kommunen, bei denen von einem größeren Infrastrukturbedarf auszugehen ist. Inwieweit der deskriptiv nahegelegte Zusammenhang zwischen Verschuldung und ÖPP-Häufigkeit bestand hat, wenn für unterschiedliche Infrastrukturbedarfe kontrolliert wird, soll im nächsten Abschnitt untersucht werden.

\section{2 Ökonometrische Modellspezifikation}

Der Effekt der kommunalen Verschuldung auf die Wahrscheinlichkeit einer Gemeinde, Infrastrukturprojekte in ÖPP zu realisieren, wird mit Hilfe verschiedener Varianten eines binären logistischen Regressionsmodells gemessen. Die allen Modellgleichungen zugrundeliegende Schätzgleichung lautet:

$$
P(y=1 x)=\alpha+\beta \text { Verschuldung }+\gamma X_{\prime}^{\prime}+\varepsilon .
$$

\footnotetext{
14 Dieser als Brechtsches Gesetz bekannte Zusammenhang wurde bereits 1932 von Arnold Brecht als „Gesetz der progressiven Parallelität von Ausgaben und Bevölkerungsmassierung“ belegt (Brecht 1932).

15 Siehe dazu ÖPP Deutschland AG (2013) sowie Grüb et al. (2016).
} 
Die abhängige Variable $y$ ist ein Dummy, der indiziert, ob eine Kommune ein oder mehrere ÖPP-Projekte durchgeführt hat. Die Variable Verschuldung ist erklärende Variable. Der Vektor $X$ enthält die Kontrollvariablen Einwohner, Einwohnerdichte und Steuereinnahmen sowie die Dummy-Variable KreisfreieStadt. Die erklärende Variable und alle Kontrollvariablen außer den Stimmanteile der bürgerlichen Parteien gehen in logarithmierter Form in die Schätzung ein.

Um mögliche unterschiedliche Wirkungsweisen der Verschuldungsformen zu berücksichtigen, werden vier verschiedene Größen für die erklärende Variable Verschuldung berücksichtigt. Modell 1 enthält die gesamte Pro-Kopf-Verschuldung, Modell 2 unterscheidet bei der Pro-Kopf-Verschuldung zwischen Investitions- und Kassenkrediten, in Modell 3 ist die Verschuldungsvariable ein Dummy, der misst, ob eine Kommune überschuldet ist oder nicht, und Modell 4 berücksichtigt die ProKopf-Nettokreditaufnahme.

Insofern höher verschuldete Kommunen eher zu ÖPP neigen, sollte der Effekt der Verschuldungsvariable in den Modellen 1-3 positiv sein. Ob auch der Effekt der Nettokreditaufnahme in Modell 4 positiv sein sollte, wenn höher verschuldete Kommunen eher ÖPP eingehen, lässt sich nicht eindeutig sagen. Die gegenwärtige Nettoneuverschuldung sollte ceteris paribus geringer sein, wenn Kommunen Infrastruktur in ÖPP bereitstellen, statt sie mit neuen Schulden zu finanzieren, die vergangene Kreditaufnahme dagegen höher. Es wird zudem getestet, ob Höhe oder Änderung der Verschuldung überschuldungsspezifische Effekte aufweisen. Dazu werden entsprechende Interaktionseffekte des Überschuldungsdummys in Modell 5 mit der ProKopf-Verschuldung und in Modell 6 mit der Pro-Kopf-Nettokreditaufnahme gemessen. Insgesamt werden also sechs Logit-Modelle geschätzt. Für alle Schätzungen werden heteroskedastie-robuste Standardfehler verwendet.

\subsection{Ergebnisse}

Die Ergebnisse der Regressionsanalyse sind in Tab. 4 zusammengefasst. Die Verschuldungsvariablen in den Modellen 1-3 weisen zwar wie erwartet positive Koeffizienten auf, jedoch kann in keinem der untersuchten Modelle ein von Null verschiedener statistisch signifikanter Zusammenhang mit der abhängigen Variable nachgewiesen werden. Die absolute Höhe der Pro-Kopf-Verschuldung hat weder aggregiert (Modell 1), noch aufgegliedert nach Investitions- und Kassenkrediten (Modell 2) einen entsprechenden Einfluss. Auch für überschuldete Kommunen lassen sich weder ein spezifischer Effekt (Modell 3) noch Interaktionseffekte mit anderen Verschuldungsvariablen (Modelle 5 und 6) feststellen. Schließlich löst auch die Pro-KopfNettokreditaufnahme keinen signifikanten Effekt aus (Modell 4). Der Koeffizient hat hier zudem ein negatives Vorzeichen.

Die Koeffizienten der verwendeten Kontrollvariablen entsprechen dagegen weitgehend den Erwartungen. Die Koeffizienten der Einwohnerzahlen und der Steuereinnahmen sind positiv und in allen Modellen auf dem $5 \%$-Niveau signifikant von Null verschieden. Der Effekt der Einwohnerdichte in allen Modellen außer in Modell 4 statistisch insignifikant, jedoch ebenfalls positiv. Überraschend erscheint der negative Zusammenhang zwischen der abhängigen Variable und den kreisfreien Städten, der jedoch ebenfalls in allen Modellen insignifikant ist. Schließlich ist der Stimman- 
Tab. 4 Ergebnisse der logistischen Regression

\begin{tabular}{|c|c|c|c|c|c|c|}
\hline & \multicolumn{6}{|c|}{ Abhängige Variable } \\
\hline & Modell 1 & Modell 2 & Modell 3 & Modell 4 & Modell 5 & Modell 6 \\
\hline Verschuldung & $\begin{array}{l}0,15 \\
(0,22)\end{array}$ & - & - & - & - & - \\
\hline Investitionskredite & - & $\begin{array}{l}0,17 \\
(0,22)\end{array}$ & - & - & - & - \\
\hline Kassenkredite & - & $\begin{array}{l}0,06 \\
(0,07)\end{array}$ & - & - & - & - \\
\hline Überschuldet & - & - & $\begin{array}{l}0,41 \\
(0,55)\end{array}$ & - & - & - \\
\hline Nettokreditaufnahme & - & - & - & $\begin{array}{l}-0,07 \\
(0,32)\end{array}$ & - & - \\
\hline $\begin{array}{l}\text { Verschuldung* } \\
\text { Überschuldet }\end{array}$ & - & - & - & - & $\begin{array}{l}0,05 \\
(0,07)\end{array}$ & - \\
\hline $\begin{array}{l}\text { Nettokreditaufnahme } \\
\text { * Überschuldet }\end{array}$ & - & - & - & - & - & $\begin{array}{l}0,07 \\
(0,09)\end{array}$ \\
\hline Einwohner & $\begin{array}{l}0,75 * * \\
(0,32)\end{array}$ & $\begin{array}{l}0,76^{* *} \\
(0,32)\end{array}$ & $\begin{array}{l}0,79 * * \\
(0,33)\end{array}$ & $\begin{array}{l}0,77 * * \\
(0,33)\end{array}$ & $\begin{array}{l}0,79 * * \\
(0,33)\end{array}$ & $\begin{array}{l}0,79 * * \\
(0,33)\end{array}$ \\
\hline Einwohnerdichte & $\begin{array}{l}0.59 \\
(0,37)\end{array}$ & $\begin{array}{l}0,55 \\
(0,37)\end{array}$ & $\begin{array}{l}0,57 \\
(0,38)\end{array}$ & $\begin{array}{l}0,62 * \\
(0,38)\end{array}$ & $\begin{array}{l}0,58 \\
(0,38)\end{array}$ & $\begin{array}{l}0,57 \\
(0,38)\end{array}$ \\
\hline Kreisfreie Stadt & $\begin{array}{l}-0,96 \\
(0,70)\end{array}$ & $\begin{array}{l}-0,98 \\
(0,71)\end{array}$ & $\begin{array}{l}-1,01 \\
(0,72)\end{array}$ & $\begin{array}{l}-0,91 \\
(0,70)\end{array}$ & $\begin{array}{l}-1,01 \\
(0,72)\end{array}$ & $\begin{array}{l}-1,02 \\
(0,72)\end{array}$ \\
\hline Steuereinnahmekraft & $\begin{array}{l}1,36 * * \\
(0,63)\end{array}$ & $\begin{array}{l}1,43^{* * *} \\
(0,61)\end{array}$ & $\begin{array}{l}1,32 * * \\
(0,57)\end{array}$ & $\begin{array}{l}1,12 \\
(0,69)\end{array}$ & $\begin{array}{l}1,31 * * \\
(0,57)\end{array}$ & $\begin{array}{l}1,33 * * \\
(0,57)\end{array}$ \\
\hline $\begin{array}{l}\text { Stimmanteil } \\
\text { CDU/FDP }\end{array}$ & $\begin{array}{l}1,20 \\
(2,03)\end{array}$ & $\begin{array}{l}1,46 \\
(2,01)\end{array}$ & $\begin{array}{l}0,90 \\
(1,85)\end{array}$ & $\begin{array}{l}0,67 \\
(1,89)\end{array}$ & $\begin{array}{l}0,88 \\
(1,85)\end{array}$ & $\begin{array}{l}0,92 \\
(1,85)\end{array}$ \\
\hline
\end{tabular}

Alle Variablen sind logarithmiert. Konstanten sind enthalten aber nicht ausgewiesen Es werden heteroskedastie-robuste Standardfehler verwendet ${ }^{*} p<0,1 ;{ }^{* *} p<0,05 ;{ }^{* * *} p<0,01$

teil bürgerlichen Parteien zwar wie erwartet positiv, aber in keinem der sechs Modell statistisch signifikant.

In Tab. 5 werden die durchschnittlichen marginalen Effekte der geschätzten Modelle dargestellt. Marginale Effekte werden durch nichtlineare Funktionen der geschätzten Koeffizienten und der jeweiligen Variablenausprägungen bestimmt. Je nach Verteilung der Variablenausprägungen können die marginalen Effekte daher von der Koeffizientenschätzung abweichende Signifikanzniveaus aufweisen. ${ }^{16}$ Im vorliegenden Modell weisen die marginalen Effekte aber gegenüber den Koeffizienten, mit Ausnahme der zuvor insignifikanten Kontrollvariablen, vergleichbare Signifikanzniveaus auf.

Die marginalen Effekte erlauben eine direkte Interpretation der Effektgrößen. Eine $10 \%$ höhere Pro-Kopf-Verschuldung geht im Durchschnitt mit einer 0,10 Prozentpunkte höheren Wahrscheinlichkeit einher, dass eine Kommune ÖPP eingeht. Die Effekte einer 10-prozentigen Erhöhung von Investitions- und Kassenkrediten

16 Im Detail siehe zu den marginalen Effekten in Logit-Modellen Dowd et al. (2014). 
Tab. 5 Durchschnittliche marginale Effekte der logistischen Regression

\begin{tabular}{|c|c|c|c|c|c|c|}
\hline & \multicolumn{6}{|c|}{ Abhängige Variable } \\
\hline & Modell 1 & Modell 2 & Modell 3 & Modell 4 & Modell 5 & Modell 6 \\
\hline Verschuldung & $\begin{array}{l}0,010 \\
(0,014)\end{array}$ & - & - & - & - & - \\
\hline Investitionskredite & - & $\begin{array}{l}0,012 \\
(0,014)\end{array}$ & - & - & - & - \\
\hline Kassenkredite & - & $\begin{array}{l}0,004 \\
(0,005)\end{array}$ & - & - & - & - \\
\hline Überschuldet & - & - & $\begin{array}{l}0,033 \\
(0,052)\end{array}$ & - & - & - \\
\hline Nettokreditaufnahme & - & - & - & $\begin{array}{l}-0,005 \\
(0,022)\end{array}$ & - & - \\
\hline $\begin{array}{l}\text { Verschuldung* } \\
\text { Überschuldet }\end{array}$ & - & - & - & - & $\begin{array}{l}0,003 \\
(0,005)\end{array}$ & - \\
\hline $\begin{array}{l}\text { Nettokreditaufnahme } \\
\text { *Überschuldet }\end{array}$ & - & - & - & - & - & $\begin{array}{l}0,005 \\
(0,006)\end{array}$ \\
\hline Einwohner & $\begin{array}{l}0,051^{* *} \\
(0,024)\end{array}$ & $\begin{array}{l}0,051^{* *} \\
(0,024)\end{array}$ & $\begin{array}{l}0,055^{* *} \\
(0,024)\end{array}$ & $\begin{array}{l}0,054^{* *} \\
(0,024)\end{array}$ & $\begin{array}{l}0,055^{* *} \\
(0,024)\end{array}$ & $\begin{array}{l}0,055^{* *} \\
(0,024)\end{array}$ \\
\hline Einwohnerdichte & $\begin{array}{l}0,041^{*} \\
(0,024)\end{array}$ & $\begin{array}{l}0,037 \\
(0,024)\end{array}$ & $\begin{array}{l}0,040 \\
(0,026)\end{array}$ & $\begin{array}{l}0,044^{*} \\
(0,025)\end{array}$ & $\begin{array}{l}0,040 \\
(0,026)\end{array}$ & $\begin{array}{l}0,040 \\
(0,026)\end{array}$ \\
\hline Kreisfreie Stadt & $\begin{array}{l}-0,047^{* *} \\
(0,023)\end{array}$ & $\begin{array}{l}-0,046^{* *} \\
(0,023)\end{array}$ & $\begin{array}{l}-0,049^{* *} \\
(0,023)\end{array}$ & $\begin{array}{l}-0,046^{*} \\
(0,024)\end{array}$ & $\begin{array}{l}-0,049^{* *} \\
(0,023)\end{array}$ & $\begin{array}{l}-0,049^{* *} \\
(0,023)\end{array}$ \\
\hline Steuereinnahmekraft & $\begin{array}{l}0,093^{* *} \\
(0,044)\end{array}$ & $\begin{array}{l}0,096^{* *} \\
(0,042)\end{array}$ & $\begin{array}{l}0,092^{* *} \\
(0,042)\end{array}$ & $\begin{array}{l}0,079 \\
(0,050)\end{array}$ & $\begin{array}{l}0,092^{* *} \\
(0,042)\end{array}$ & $\begin{array}{l}0,093^{* *} \\
(0,042)\end{array}$ \\
\hline $\begin{array}{l}\text { Stimmanteil } \\
\text { CDU/FDP }\end{array}$ & $\begin{array}{l}0,082 \\
(0,140)\end{array}$ & $\begin{array}{l}0,098 \\
(0,136)\end{array}$ & $\begin{array}{l}0,063 \\
(0,131)\end{array}$ & $\begin{array}{l}0,047 \\
(0,134)\end{array}$ & $\begin{array}{l}0,061 \\
(0,131)\end{array}$ & $\begin{array}{l}0,064 \\
(0,131)\end{array}$ \\
\hline
\end{tabular}

Alle Variablen sind logarithmiert

Konstanten sind enthalten aber nicht ausgewiesen ${ }^{*} p<0,1 ;{ }^{* *} p<0,05 ;{ }^{* * *} p<0,01$

sowie Pro-Kopf-Nettokreditaufnahme bewegen sich mit 0,12 Prozentpunkten, 0,04 Prozentpunkten und minus 0,05 Prozentpunkten in einer ähnlichen Größenordnung. Gleiches gilt für die überschuldungsspezifischen Effekte von Pro-Kopf-Verschuldung und Pro-Kopf-Nettokreditaufnahme mit 0,03 und 0,05 Prozentpunkten. Bei der Interpretation des Effekts der Überschuldung ist zu beachten, dass sich der Effekt hier nicht auf die relative Änderung einer kontinuierlichen Variable, sondern auf den gesamten Einfluss einer binären Variable bezieht. Gilt eine Kommune als überschuldet, weist sie durchschnittlich eine 3,3 Prozentpunkte höhere Wahrscheinlichkeit auf ÖPP einzugehen. Insgesamt bedeuten die Ergebnisse, dass der Effekt der Verschuldung einer Kommune auf die Wahrscheinlichkeit eine ÖPP einzugehen nicht nur statistisch, sondern auch ökonomisch insignifikant ist, d. h. quantitativ unbedeutend.

Die geschätzten durchschnittlichen Effekte der Kontrollvariablen sind dagegen größtenteils nicht nur statistisch signifikant, sondern auch um ein Vielfaches größer als die der Verschuldungsvariablen. Beispielsweise geht eine zehnprozentige Erhöhung der Einwohnerzahl mit einer durchschnittlich 0,51 bis 0,55 Prozentpunk- 
te höheren Wahrscheinlichkeit einher, dass eine Kommune Infrastrukturprojekte in ÖPP realisiert.

Insgesamt lässt sich ein Effekt der Verschuldung darauf, dass eine Kommune ein oder mehrere ÖPP-Projekte durchführt, empirisch nicht belegen. Zwar neigen ÖPPKommunen zu höherer Verschuldung. Der Zusammenhang zwischen Verschuldung und ÖPP-Häufigkeit verschwindet aber, wenn für weitere Fiskal- und Strukturdaten kontrolliert wird. Natürlich kann trotz fehlender statistischer Signifikanz ein Zusammenhang zwischen Verschuldung und ÖPP-Häufigkeit nicht ausgeschlossen werden. Allerdings deuten die Größen der geschätzten Parameter darauf hin, dass ein Einfluss, so er denn besteht, ökonomisch von nur geringer Bedeutung sein dürfte.

\section{Fazit}

Der vorliegende Beitrag findet keine belastbare empirische Evidenz für die gelegentlich geäußerte Vermutung, dass sich höher verschuldete Gebietskörperschaften stärker in ÖPP engagieren. Zwar legt eine isolierte Betrachtung von ÖPP-Projekten und Verschuldung von Kommunen in Nordrhein-Westfalen nahe, dass ein solcher Zusammenhang besteht. Dieser Zusammenhang hält aber einer multivariaten Betrachtung nicht stand.

In dem Maße, in dem das vorliegende Ergebnis belastbar ist, enthält es eine gute Nachricht. Es legt nämlich nahe, dass die Kommunen in Nordrhein-Westfalen ÖPP nicht dazu missbrauchen, das Ausmaß öffentlicher Finanzierungsverpflichtungen zu verschleiern. Allerdings basiert die empirische Analyse auf einem unvollständigen Datensatz, weil ÖPP-Projekte in Deutschland nur lückenhaft erfasst werden.

Durch die Zusammenführung von PPP-Projektdatenbank und Schuldenstatistik konnten zwar einige Unzulänglichkeiten in den Datensätzen bereinigt werden. Eine systematische Erfassung aller ÖPP-Projekte im Beobachtungszeitraum stellt der entwickelte Datensatz aber nicht dar. Insbesondere kann nicht ausgeschlossen werden, dass einzelne ÖPP-Projekte nordrhein-westfälischer Kommunen unberücksichtigt geblieben sind. Es ist deshalb dringend angeraten, dass ÖPP-Projekte in Zukunft systematisch und vollständig erfasst werden und zwar sowohl hinsichtlich des gesamten Finanzierungsvolumens, als auch hinsichtlich der zeitlichen Struktur der Vertragslaufzeiten und der Zahlungsströme. Nur so wird eine umfassende evidenzbasierte Bewertung von ÖPP möglich.

Funding Open Access funding provided by Projekt DEAL.

Open Access Dieser Artikel wird unter der Creative Commons Namensnennung 4.0 International Lizenz veröffentlicht, welche die Nutzung, Vervielfältigung, Bearbeitung, Verbreitung und Wiedergabe in jeglichem Medium und Format erlaubt, sofern Sie den/die ursprünglichen Autor(en) und die Quelle ordnungsgemäß nennen, einen Link zur Creative Commons Lizenz beifügen und angeben, ob Änderungen vorgenommen wurden.

Die in diesem Artikel enthaltenen Bilder und sonstiges Drittmaterial unterliegen ebenfalls der genannten Creative Commons Lizenz, sofern sich aus der Abbildungslegende nichts anderes ergibt. Sofern das betreffende Material nicht unter der genannten Creative Commons Lizenz steht und die betreffende Handlung nicht nach gesetzlichen Vorschriften erlaubt ist, ist für die oben aufgeführten Weiterverwendungen des Materials die Einwilligung des jeweiligen Rechteinhabers einzuholen. 
Weitere Details zur Lizenz entnehmen Sie bitte der Lizenzinformation auf http://creativecommons.org/ licenses/by/4.0/deed.de.

\section{Literatur}

Albalate, D., Bel, G., \& Geddes, R.R. (2015). The determinants of contractual choice for private involvement in infrastructure projects. Public Money and Management, 35(1), 87-94.

Auriol, E., \& Picard, P. M. (2013). A theory of BOT concession contracts. Journal of Economic Behavior and Organization, 89, 187-209.

Brecht, A. (1932). Internationaler Vergleich der öffentlichen Ausgaben. Leipzig: Teubner.

Buso, M., Marty, F., \& Tran, P.T. (2017). Public-private partnerships from budget constraints: looking for debt hiding? International Journal of Industrial Organization, 51, 56-84.

Destatis (2019). Pressemitteilung Nr. 352 vom 12. September 2019. Wiesbaden: Destatis.

Dowd, B.E., Greene, W.H., \& Norton, E.C. (2014). Computation of standard errors. Health Services Research, 49(2), 731-750.

Easterly, W. (1999). When is fiscal adjustment an illusion? Economic Policy, 14(28), 56-86.

Eurostat (2014). Europäisches System Volkswirtschaftlicher Gesamtrechnungen - ESVG 2010

Funke, K., Irwin, T., \& Rial, I. (2013). Budgeting and reporting for public-private partnerships. Bd. 7. Paris: OECD Publishing.

Grüb, B., Theuvsen, L., Emmann, C.H., \& Schaper, C. (2016). Öffentlich-Private Partnerschaften in deutschen Kommunen - Erfahrungen und Einstellungen kommunaler Entscheidungsträger. In Sonderheft ZÖGU - Öffentlich-Private Partnerschaften - Potentiale und Probleme (S. 40-59).

Hesse, M., Lück, O., Redlich, M., \& Rottmann, O. (2013). Empirischer Vergleich von KBV und ÖPP - Studie zu Beschaffungsmethoden der öffentlichen Hand vor dem Hintergrund des Gemeinsamen Erfahrungsberichts der Rechnungshöfe

Landeswahlleiter, N.R.W. (2014). Ergebnisse der Kommunalwahlen 2014. https://www.wahlergebnisse. nrw/kommunalwahlen/2014/index.html. Zugegriffen: 18. März. 2020.

Ministerium für Inneres und Kommunales NRW. (2018). Haushaltsstatus der Gemeinden in NordrheinWestfalen

Mühlenkamp, H. (2014). Public-Private Partnerships and government debt. CESifo DICE Report, 12(3), 24-30.

ÖPP Deutschland AG (Partnerschaft Deuschtschland). (2013). Transparenz bei ÖPP Projekten. ÖPPSchriftenreihe, Bd. 10. Berlin.

ÖPP Deutschland AG (Partnerschaft Deutschland) (2011). Öffentlich-Private Partnerschaften im Bereich von Kindergärten und Kindertagesstätten. ÖPP-Schriftenreihe, Bd. 3. Berlin: ÖPP.

Partnerschaft Deuschtschland (2020). PPP-Projektdatenbank. https://www.ppp-projektdatenbank.de/. Zugegriffen: 17. Febr. 2020.

Rappen, H. (2017). Stärkungspakt Stadtfinanzen - Weg aus der Schuldenfalle oder gekaufte Zeit? RWI Materialien, 120. https://doi.org/10.4419/86788829.

Russo, F. A., \& Zampino, R. (2010). Machiavellian PPP?-evidence from Italian local government's projects for public services. IV International Public Procurement Conference Proceedings.

Statistische Ämter (2019). Regionaldatenbank Deutschland. https://www.regionalstatistik.de/.Zugegriffen: 17. Febr. 2020.

Statistisches Landesamt, N. R. W. (2016). Kommunale Schuldenstatisik NRW

Wigger, B. U. (2017). Vor- und Nachteile Öffentlich-Privater Partnerschaften. List Forum für Wirtschaftsund Finanzpolitik, 42(4), 395-421. 